\title{
Transcatheter Tricuspid Valve Replacement and Repair: Pooled Analysis of the Outcomes and Complications of Novel Emerging Treatments

\author{
Yaser Khalid, DO ${ }^{1}$ (D) Neethi Dasu, DO ${ }^{1^{*}}$ (D) and Kirti Dasu, BA ${ }^{2}$ (D)
}

${ }^{1}$ Division of Internal Medicine, Rowan University School of Medicine at Jefferson Health System, Stratford, New Jersey, USA

${ }^{2}$ Division of Biology, Syracuse University, Syracuse, USA

*Corresponding author: Dr. Neethi Dasu, Rowan School of Medicine, Jefferson Health System, One Medical Center Dr, UDP Suite 2300 Stratford, NJ 08084, USA, Tel: 6093503615

\begin{abstract}
Background: Tricuspid regurgitation is a poor prognostic marker of end-stage heart failure. Treatment options for severe tricuspid regurgitation are currently limited because these procedures have the highest mortality rates of all valve procedures. Isolated tricuspid valve surgery is reserved for rare cases during surgical viability evaluation with repair favored over tricuspid valve replacement, owing to the slightly more favorable mortality and downstream complication associated with the latter. Transcatheter tricuspid valve repair or replacement (collectively, TVTT), on the other hand, are extremely promising interventions due to the minimally invasive nature of these treatments. Multiple devices are currently being developed and tested for TTVT to offer surgeons a safer alternative than the aforementioned traditionally high-risk surgical procedures.
\end{abstract}

Objective: The aim of this study is to examine the complications of these procedures from day 0 of procedure to up to 1 year of follow up.

Methods: Six studies were included, 3 were registries (TriVALVE, TRILUMINATE, TRAMI), surveying a total of 336 patients. Complications following the procedures were pooled and analyzed.

Results: From procedure to up to 1 year, there was $14.3 \%$ mortality (49/342), $0.3 \%$ incidence of myocardial infarction and TIA/stroke (1/342), $2 \%$ device embolization and/ or leaflet detachment (7/342), 5.2\% major/minor bleeding (18/342), 0.9\% AKI (3/342), 1.1\% infections and arrhythmias $(4 / 342)$.
Conclusions: Despite a narrow sample size due to the novelty of these procedures and varying lengths of follow-up (30 days to 1 year), transcatheter tricuspid valve repair and replacement prove to be promising interventions. Mortality was significant at $14.6 \%$, but patients with severe tricuspid regurgitation have a poor prognosis overall without intervention along with higher comparative mortality rates. Research aimed at further investigating TVTT procedures and prospective clinical trials to establish these treatments as mainstays for severe tricuspid regurgitation is necessary.

\section{Keywords}

Tricuspid valve, Tricuspid regurgitation, Transcatheter procedures, Mortality, Heart failure

\section{Introduction}

Tricuspid regurgitation (TR) is a valvular disease that can occur from a multitude of causes, including right ventricle (RV) and tricuspid annular dilatation, often late after left-sided valve surgery and secondary to left-sided heart failure $[1,2]$. There is a decent prevalence of TR worldwide that have clinically relevant TR: 1.6 million patients in the USA and 3.0 million in Europe [3]. Moderate-to-severe TR significantly increases mortality for a majority of patients, independently of other comorbidities [1,4]. The number of cases of TR has been increasing with a greater number of aging populations. The economic, physiologic and patient burden has become noticeable and there has been an increase in surgical intervention for TR [5].

Citation: Khalid Y, Dasu N, Dasu K (2020) Transcatheter Tricuspid Valve Replacement and Repair: Pooled Analysis of the Outcomes and Complications of Novel Emerging Treatments. Int J Clin Cardiol 7:179. doi.org/10.23937/2378-2951/1410179

Accepted: May 30, 2020; Published: June 01, 2020

Copyright: (c) 2020 Khalid Y, et al. This is an open-access article distributed under the terms of the Creative Commons Attribution License, which permits unrestricted use, distribution, and reproduction in any medium, provided the original author and source are credited. 
Currently, few treatment options are available for TR. Management has always been very difficult due to the complex hemodynamics that must be managed for moderate-to-severe TR. Most guideline-directed medical treatment is limited to diuretics for improvement of symptoms with little impact on morbidity and mortality [6]. Surgical repair or replacement of TR is rarely performed because there is significant mortality, as high as $18 \%$ and $33 \%$ respectively $[1,7]$. Furthermore, more patients with significant TR undergo repair rather than replacement; however, outcomes are substandard as a large number of patients, approximately $25 \%$, develop moderate or severe TR within 5 years of repair [5]. Recently, due to the risk of surgery, transcatheter repair and replacement have emerged as novel and promising treatment options.

Although there is limited evidence for the use of these treatments, current methods of TR replacement include: leaflet and coaptation devices for edge-to-edge repair and valve replacement, valve replacement, both in the heterotopic (caval valve implantation to reduce the backflow in the venous system) and the orthotropic positions [8]. There is no consensus on whether it is more beneficial for patients to undergo repair or replacement of the tricuspid valve nor on the type of device to use.

We aim to study clinical outcomes of patients who undergo transcatheter repair or replacement of tricuspid valves with moderate or severe TR from the time of procedure to up to one year of follow-up. The primary endpoint studied was mortality. The secondary endpoints analyzed were cardiovascular events, transient ischemic attack (TIA)/stroke, device embolization and leaflet dysfunction, major and minor bleeding events, acute kidney injury (AKI), infection and arrhythmias.

\section{Methods}

\section{Search methods and study selection}

An electronic database search was conducted through MEDLINE/PUBMED, EBSCO, EMBASE, Thomson Reuters' Web of Science, the Cochrane Library, Google Scholar, Central Register of Controlled Trials (CENTRAL) and ClinicalTrials.gov in April 20, 2020 and updated in May 01, 2020. Standardized methods were utilized for searching the following keywords: "tricuspid," "tricuspid valve," "tricuspid valve regurgitation," "mild tricuspid valve regurgitation," "moderate tricuspid valve regurgitation," "severe tricuspid valve regurgitation," "tricuspid valve repair," "tricuspid valve replacement," "surgical tricuspid valve replacement," "transcatheter tricuspid valve replacement," "surgical tricuspid valve repair," "transcatheter tricuspid valve repair." Extensive hand searching was conducted by two authors by screening references or included studies as well as review articles for additional citations.

Additionally, both titles and abstracts were screened for the exclusion of unrelated articles. The references of all included articles were reviewed for additional relevant articles. No language restrictions were assumed for the included articles.

We considered eligible all prospective controlled trials examining outcomes following transcatheter tricuspid valve replacement or repair. They were considered for inclusion irrespective of patients' baseline conditions, background therapy, study follow-up or language of publication. There were no trials that compared transcatheter versus surgical tricuspid valve replacement or repair. There were no trials that compared transcatheter repair versus transcatheter valve replacement.

Whenever possible the adverse events were reported according to the Common Terminology Criteria for Adverse Events (CTCAE). The records retrieved through electronic database search were screened independently by 2 authors.

\section{Data extraction}

Suitable studies were evaluated for the inclusion in the review through full-text assessment. Study selection and data extraction were performed independently. If different data were available for the same trial, we considered the most recent report or the updated data from ClinicalTrials.gov. Using a data extraction table on Microsoft Excel, the required information from each article, including first author's name, publication year, study design, sample size, outcomes from transcatheter tricuspid valve replacement or repair, and incidence of complications, was recorded. The table was completed by the first author and verified by an additional member of the study team.

Study characteristics and results were extracted independently into a standardized form. Disagreements throughout this process were resolved by consensus. In the case where the needed data was not directly found in the published articles, we obtained such data from the authors through response letters/e-mail or via reviewing their supplemental reports.

\section{Pooled-analysis}

The data statistical analysis was performed through the Hartung-Knapp-Sidik-Jonkman (HKSJ) method in Comprehensive Meta-analysis software for single events in a single sample size. A secondary analysis was performed using the Mantel-Haenszel method through the MedCalc software.

The total number of each event from every study was taken and then added together with the total number of events from all of the other studies for an aggregate number of events. This was then divided by the total number of patients to produce a total percentage of each complication for all six of the studies. The Z-test was used for calculating the confidence interval and $p$-values for each mean. 
All statistical tests were two-tailed, and the type I error rate was set at $5 \%$. Only two-sided tests with a significance level of 0.05 were used. Confidence intervals (Cls) of individual studies were determined or approximated from the available data. A sensitivity analysis was also conducted, in which each study was omitted in turn. Heterogeneity was described using the $1^{\wedge} 2$ statistic, which was quantified as low $(<25 \%)$, moderate $(25 \%$ to $75 \%$ ), or high (> $75 \%$ ).

\section{Results}

\section{Baseline characteristics}

Our study population had a mean age of 76.4 years and $57 \%$ were females. The patient population's comor- bidities were the following: $76 \%$ atrial fibrillation, $30 \%$ diabetes, 92\% New York Heart Association class III/IV heart failure, $26 \%$ cardiac resynchronization therapy (CRT)/implantable cardiac defibrillator (ICD)/permanent pacemaker placement (PPM), 76\% renal disease (defined as GFR < 60). Previously, $20 \%$ of the patients had a myocardial infarction (MI) and $34 \%$ had previous valve surgery (only 4 studies reported this data). Mean EuroSCORE was 13.65 representing a significant surgical risk at baseline. The baseline echocardiograms revealed a tricuspid annular plane systolic excursion (TAPSE) of $15.7 \mathrm{~cm}$ and 3,359 ng/dL NT-proBNP.

\section{Procedures}

Current methods of tricuspid valve replacement in-

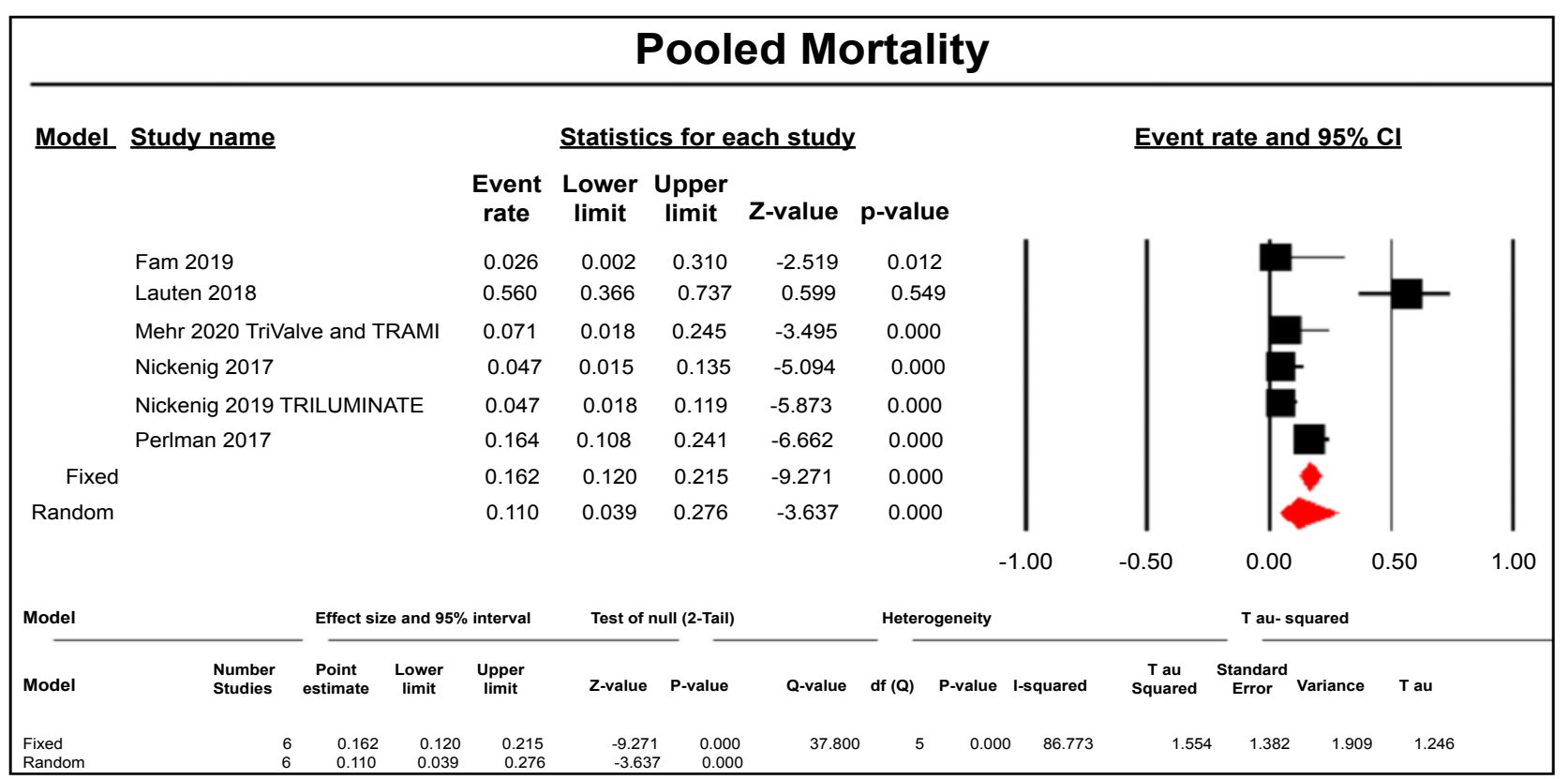

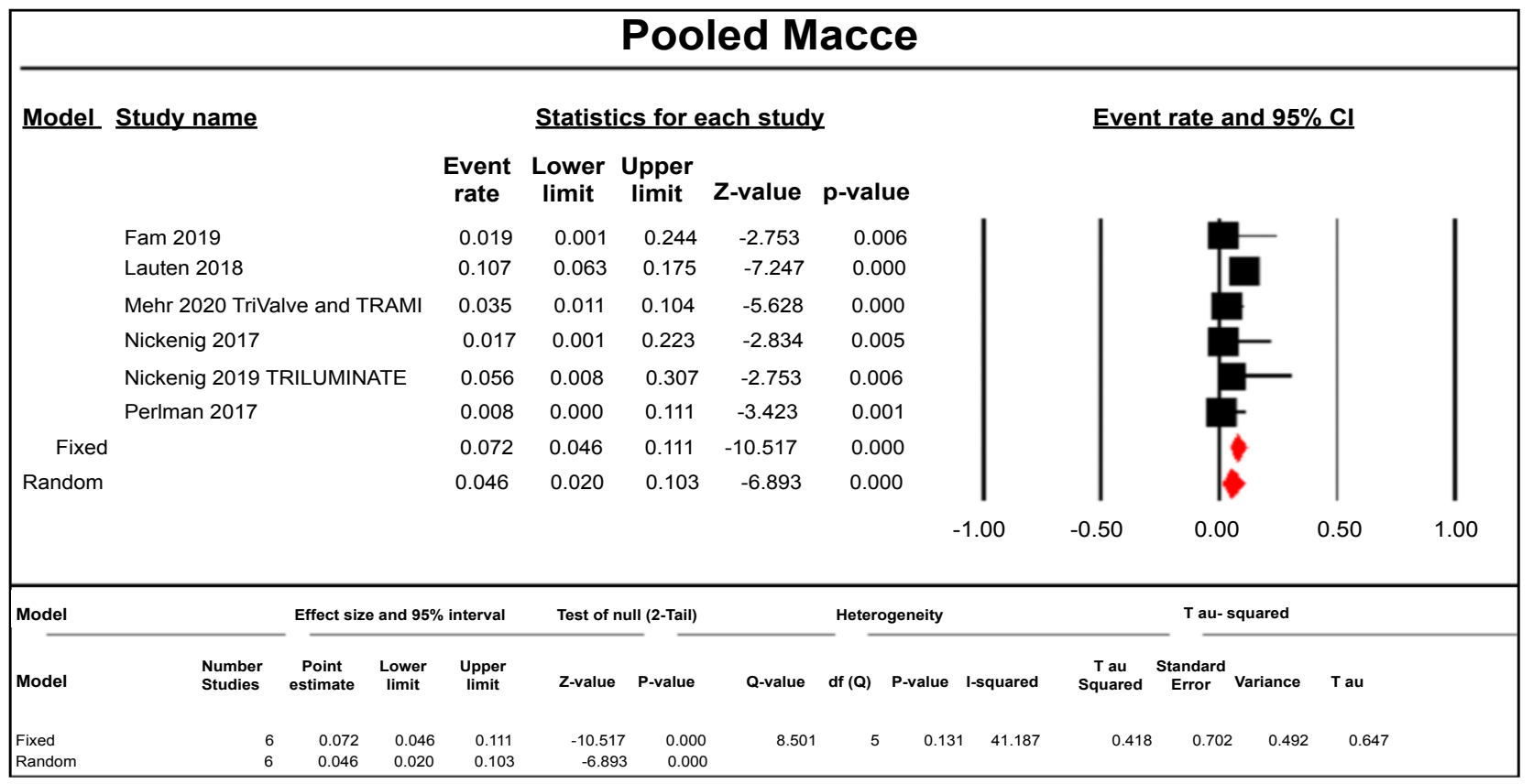

Figure 1: Pooled Mortality and MACCE following transcatheter tricuspid valve repair and replacement. The relative size of the data markers indicates the weight of the sample size from each study.

Cl: Confidence Interval; RR: Risk Ratio. 
clude the caval approach via: single (inferior vena cava) or bicaval implantation (inferior vena cava and superior vena cava) with either balloon-expandable valves (Sapien XT/3) or self-expandable valves (TricValve or Directflow) [9]. Methods for edge-to-edge repair include use of the PASCAL system (which is a configuration of two adjacent, contoured paddles and a central spacer) [10], MitraClip [11], TriClip [3,6] and FORMA system [1]. In our analyses of six studies, there was: 1 TriClip, 2 MitraClip, 1 single or bicaval valve (Sapien, TricValve, DirectFlow were all used in one study), 1 FORMA spacer, and 1 PASCAL were employed.

\section{Clinical outcomes}

From procedure to up to 1 year, there was $14.3 \%$ mortality (49/342) (Figure 1), $0.3 \%$ incidence of myocardial infarction (Figure 1) and TIA/stroke (1/342) (Figure 2), $2 \%$ device embolization and/or leaflet detachment (7/342) (Figure 2), 5.2\% major/minor bleeding (18/342) (Figure 3), 0.9\% AKI (3/342) (Figure 3), 1.1\% infections (Figure 4) and arrhythmias (4/342) (Figure 4). All findings were statistically relevant with a $p$-value $<0.05$. There was moderate heterogeneity with all of our outcomes, between 20 to $75 \%$.

\section{Discussion}

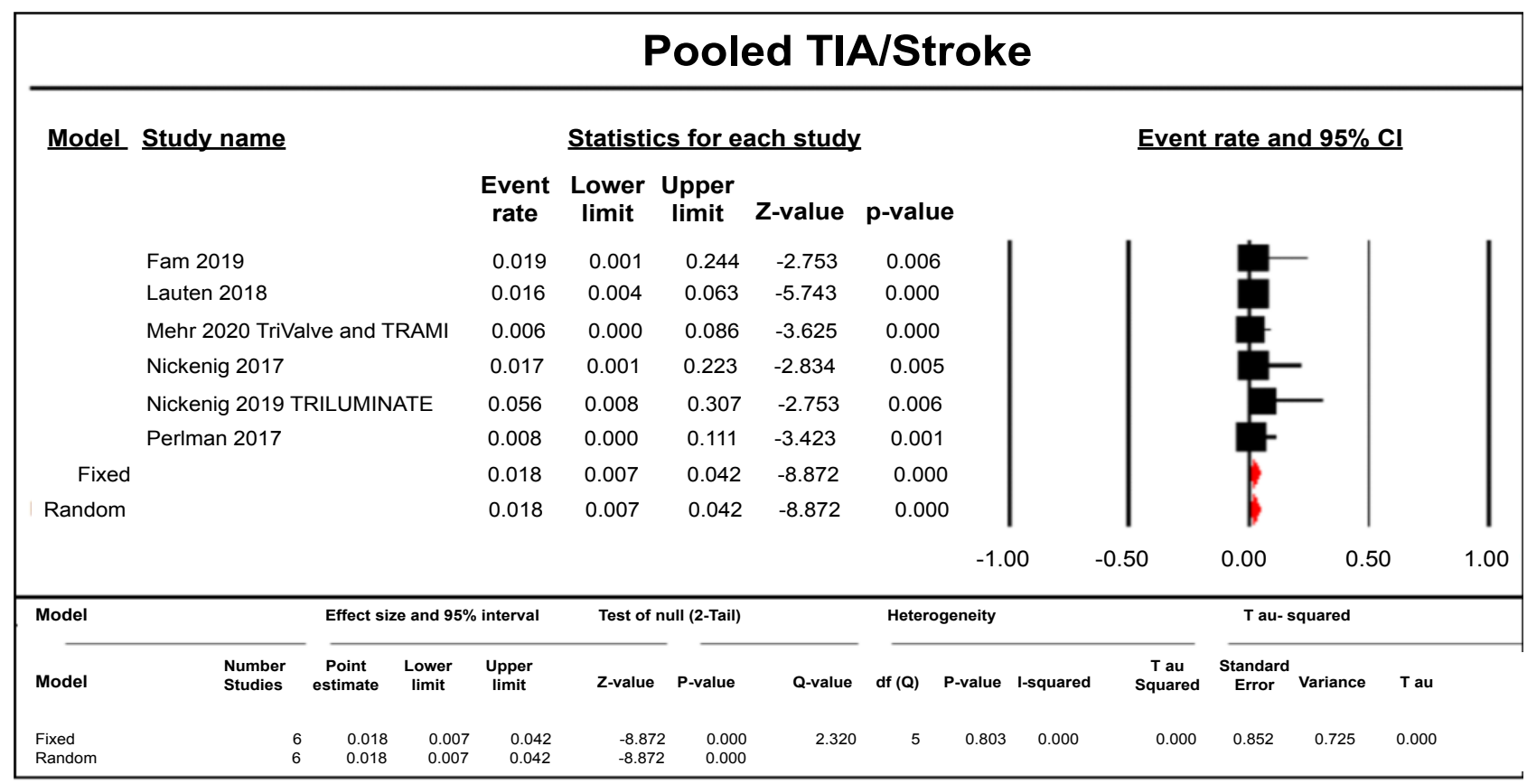

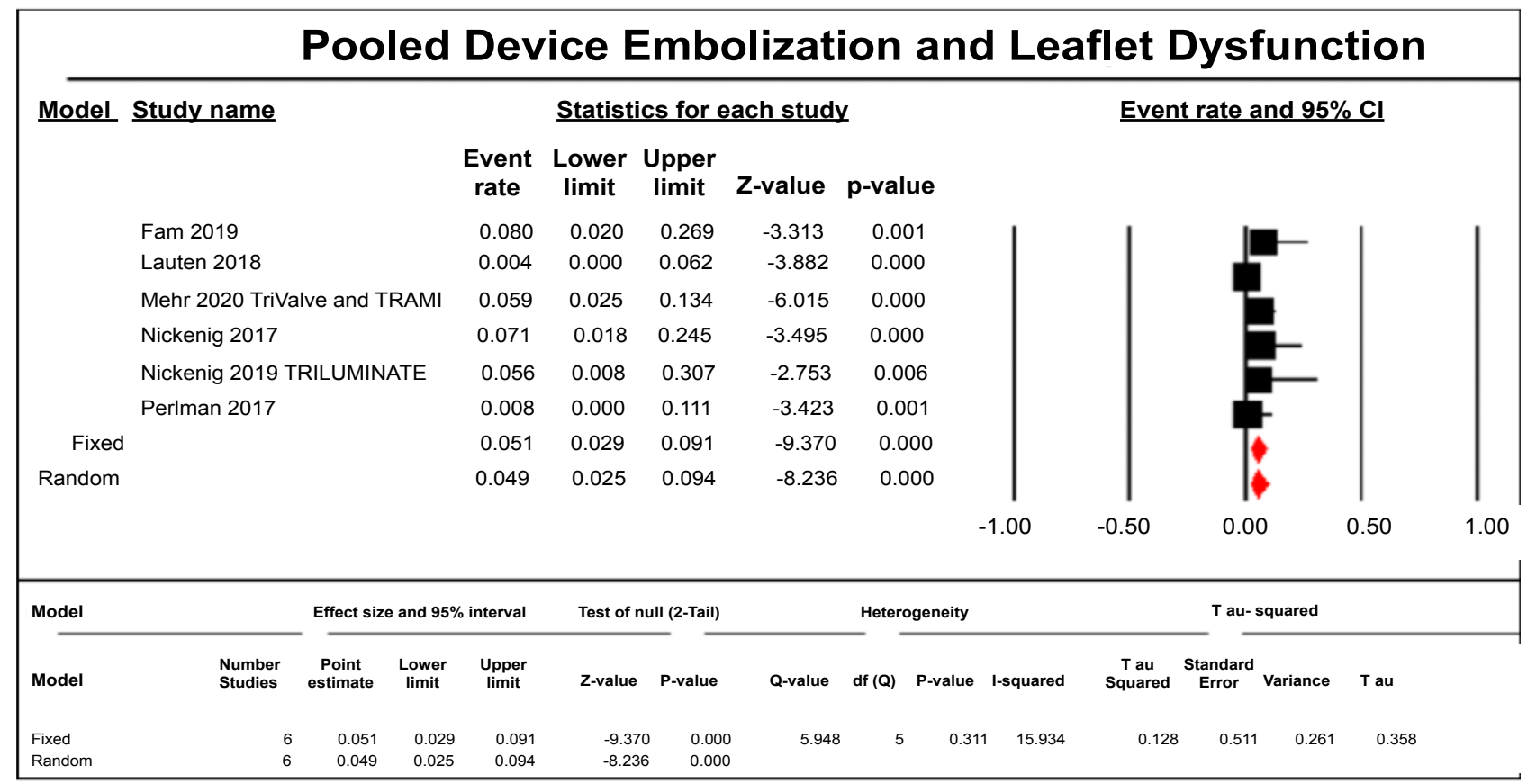

Figure 2: Pooled TIA/Stroke as well as Embolization and Leaflet Dysfunction following transcatheter tricuspid valve repair and replacement.

The relative size of the data markers indicates the weight of the sample size from each study.

Cl: Confidence Interval; RR: Risk Ratio. 


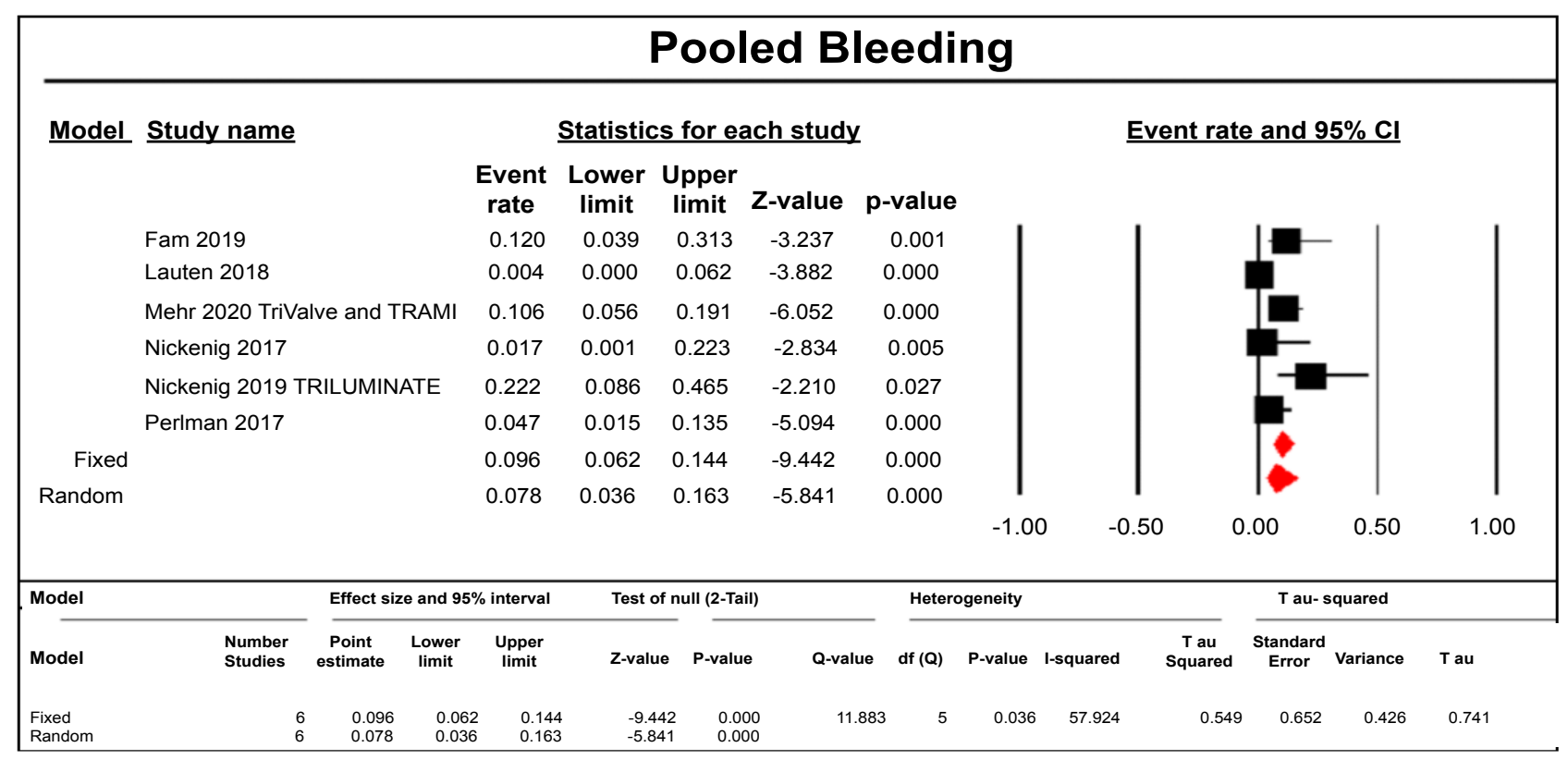

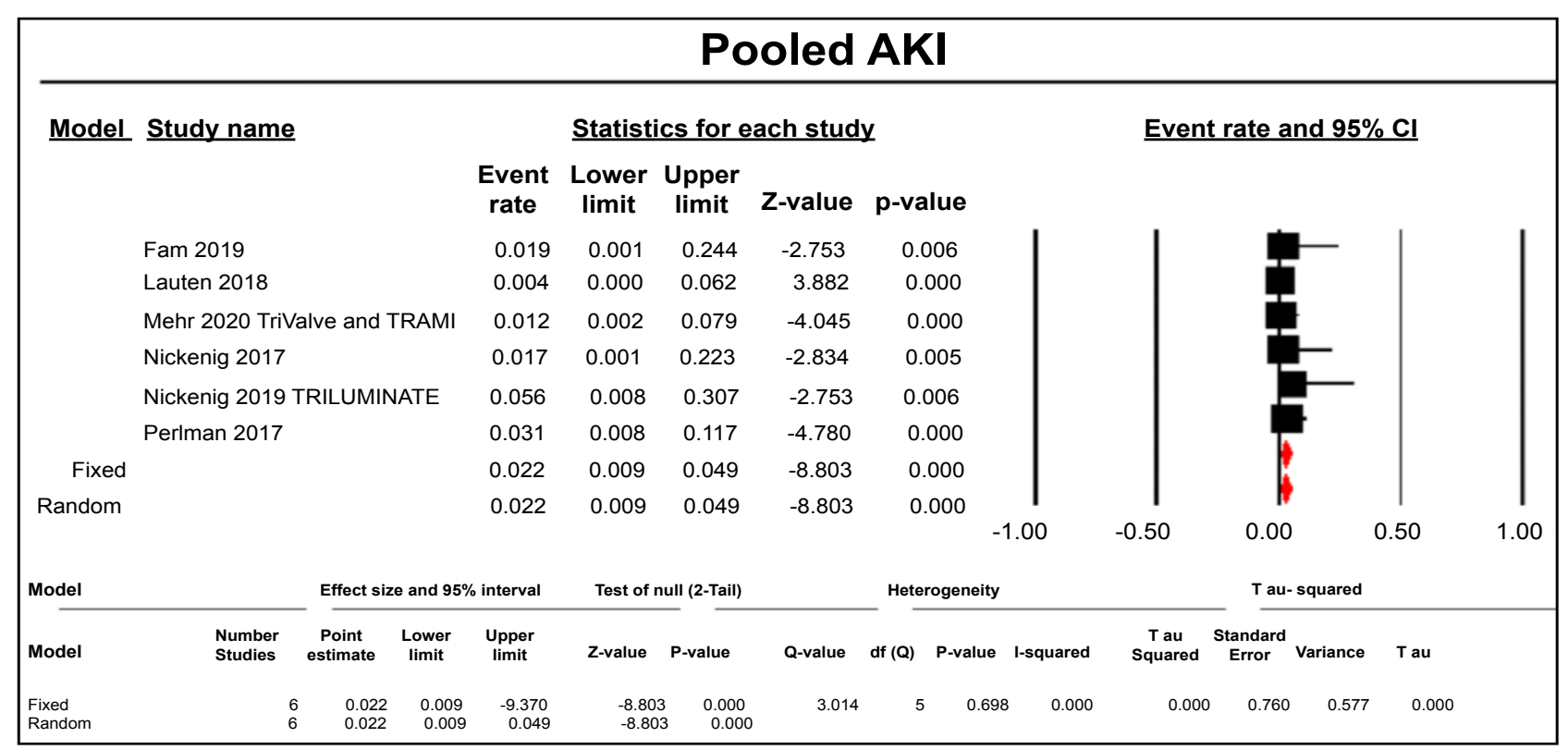

Figure 3: Pooled Major and Minor Bleeding and AKI following transcatheter tricuspid valve repair and replacement. The relative size of the data markers indicates the weight of the sample size from each study.

Cl: Confidence Interval; RR: Risk Ratio.

TR affects more than 1.5 million Americans yearly. It is a source of significant mortality and morbidity, however, this condition is insufficiently studied and under-treated [12]. There are currently three subtypes of TR, which include primary, secondary, and isolated. These classifications of TR are based on the underlying pathophysiological processes [13]. Primary TR is caused by anatomical malformations or structural abnormalities of the valve or its components while secondary TR is usually a result of left-sided heart failure [13]. Isolated TR is associated with atrial tachyarrhythmias and is an entity that can exist with normal RV pressures [13]. Then, TR is further classified into mild, moderate, and severe based on regurgitant volume across the tricuspid valve seen on echocardiography. Our study analyzed patients with moderate and severe TR.
Currently, less than one percent of patients with moderate or severe TR are intervened on surgically, with a $44 \%$ mortality noted at 10 years post-procedure $[12,14]$. Patients with moderate or severe TR also have a higher risk of all-cause mortality as well as cardiac mortality, when matched to the general population [15]. Many trials have demonstrated that even a mild reduction of TR severity, even if the remaining TR is still severe, is associated with a significant improvement of symptoms and quality of like, likely secondary to better cardiac output and renal perfusion [16]. Thus, there exists a great need for other minimally invasive approaches to tricuspid valve repair. We analyzed outcomes from six studies evaluating transcather repair or replacement of patients with severe or moderate TR. There are two main findings from our pooled analysis of six prospec- 


\section{Pooled Arrhythmias}

\begin{tabular}{|c|c|c|c|c|c|c|c|c|c|c|c|c|c|c|}
\hline \multirow[t]{8}{*}{ Model } & \multirow{2}{*}{\multicolumn{3}{|c|}{ Study name }} & \multicolumn{5}{|c|}{ Statistics for each study } & \multicolumn{6}{|c|}{ Event rate and $95 \% \mathrm{Cl}$} \\
\hline & & & & $\begin{array}{l}\text { Event } \\
\text { rate }\end{array}$ & $\begin{array}{l}\text { Lower } \\
\text { limit }\end{array}$ & $\begin{array}{l}\text { Upper } \\
\text { limit }\end{array}$ & Z-value $p$ & p-value & & & & & & \\
\hline & Fam 2019 & & & 0.019 & 0.001 & 0.244 & -2.753 & 0.006 & & & & & & \\
\hline & Lauten 2018 & & & 0.004 & 0.000 & 0.062 & -3.882 & 0.000 & & & & & & \\
\hline & Mehr 2020 TriVal & ve and $T F$ & RAMI & 0.012 & 0.002 & 0.079 & -4.405 & 0.000 & & & & & & \\
\hline & Nickenig 2017 & & & 0.017 & 0.001 & 0.223 & -2.834 & 0.005 & & & & & & \\
\hline & Nickenig 2019 TP & RILUMIN & & 0.056 & 0.008 & 0.307 & -2.753 & 0.006 & & & & & & \\
\hline & Perlman 2017 & & & 0.047 & 0.015 & 0.135 & -5.094 & 0.000 & & & & & & \\
\hline Fixed & & & & 0.028 & 0.013 & 0.059 & -8.913 & 0.000 & & & & 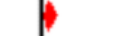 & & \\
\hline Random & & & & 0.028 & 0.013 & 0.059 & -8.913 & 0.000 & & & & 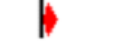 & & \\
\hline & & & & & & & & & -1. & & -0.50 & 0.00 & 0.50 & 1.00 \\
\hline Mottel & & Effect siz & $e$ and 95 & $\%$ interval & Test of & f null (2-Tail) & & Heterog & geneity & & & T au- sq & luared & \\
\hline Model & $\begin{array}{l}\text { Number } \\
\text { Studies }\end{array}$ & $\begin{array}{l}\text { Point } \\
\text { estimate }\end{array}$ & $\begin{array}{l}\text { Lower } \\
\text { limit }\end{array}$ & $\begin{array}{l}\text { Upper } \\
\text { limit }\end{array}$ & $z$-valu & ue $P$-value & Q-value & e df (Q) & P-value & I-squared & $\begin{array}{c}\text { T au } \\
\text { Squared }\end{array}$ & $\underset{\text { Error }}{\text { Standard }}$ & ariance & T au \\
\hline $\begin{array}{l}\text { Fixed } \\
\text { Random }\end{array}$ & $\begin{array}{l}6 \\
6\end{array}$ & $\begin{array}{l}0.028 \\
0.028\end{array}$ & $\begin{array}{l}0.013 \\
0.013\end{array}$ & $\begin{array}{l}0.059 \\
0.059\end{array}$ & $\begin{array}{l}-8.91 \\
-8.91\end{array}$ & $\begin{array}{l}0.000 \\
0.000\end{array}$ & 4.165 & 5 & 0.526 & 0.000 & 0.000 & 0.694 & 0.482 & 0.000 \\
\hline
\end{tabular}

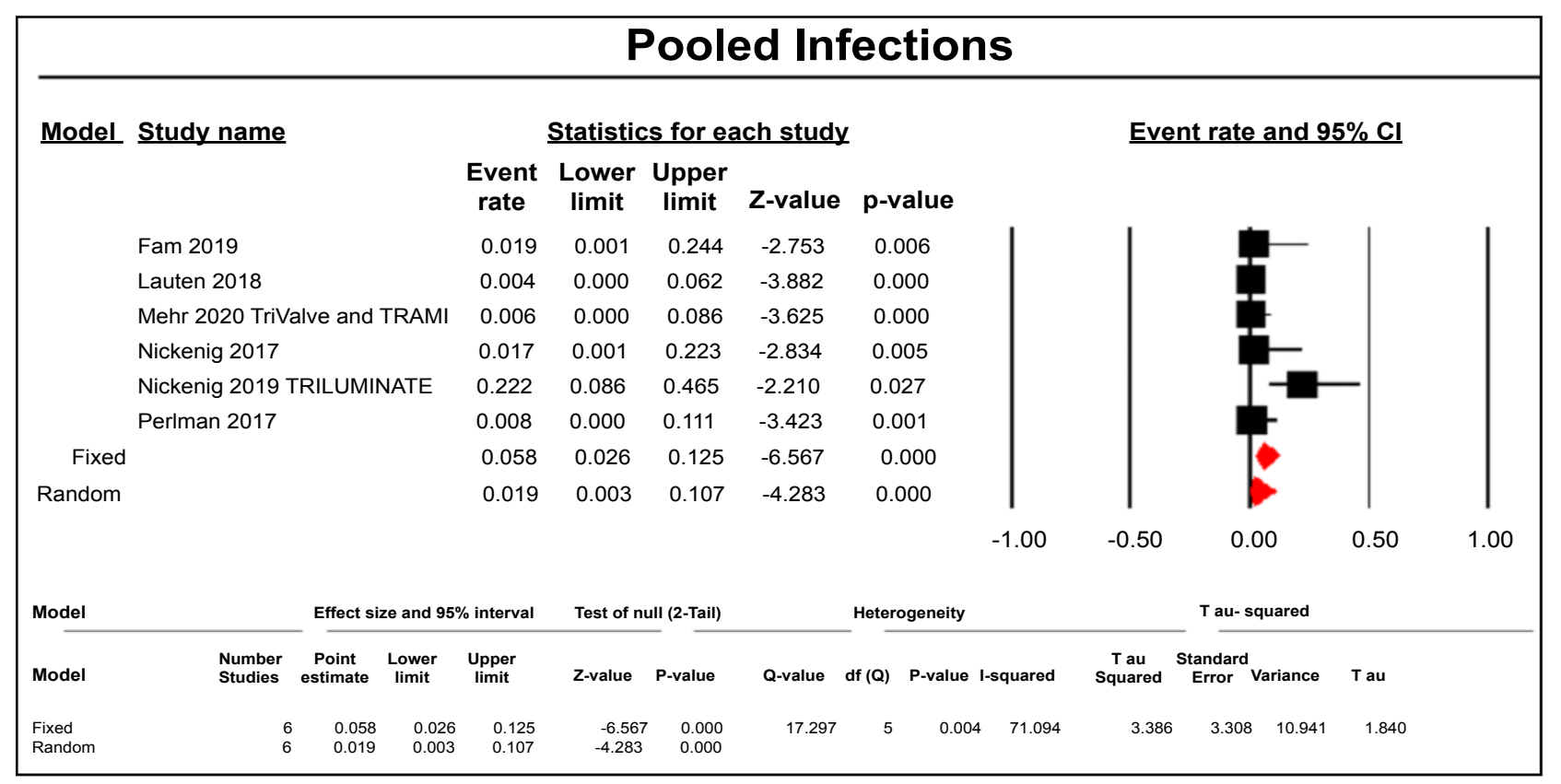

Figure 4: Pooled Arrhythmias and Infections following transcatheter tricuspid valve repair and replacement.

The relative size of the data markers indicates the weight of the sample size from each study.

Cl: Confidence Interval; RR: Risk Ratio.

tive controlled trials: mortality was significantly reduced for a patient population, which already had an extremely high baseline mortality without treatment, and the risk of complications was noted to be low.

There are currently many challenges to overcome for transcatheter tricuspid valve repair or replacement: large tricuspid annulus dimensions (usually $>40 \mathrm{~mm}$ in diameter) with a non-planar and elliptical shape; right ventricular morphology (trabeculations, muscle bands, thin apical wall); and proximity of other structures [17]. Previously, coaptation depth, a measurement of leaflet tethering, for repair or replacement was found to be the biggest factor that influenced success or failure of the intervention [18]. Our study confirms that baseline comorbidities may not impact the outcomes as much as initially thought. At baseline, most of our patients had significant comorbidities, such as atrial fibrillation, renal disease, and advanced heart failure, yet the mortality was only approximately $14 \%$ following transcatheter intervention. This further illustrates that mastering the technique of repair or replacement and intervening earlier, prior to permanent RV remodeling, may be the best method of improving patient outcomes [8].

Additionally, our studies used various devices for intervention. A majority of the devices were coaptation devices for edge-to-edge repair: FORMA, PASCAL, and MitraClip. The MitraClip is well known for the repair of functional mitral regurgitation. The FORMA is a valve 
spacer, which is positioned into the regurgitant orifice in order to create a platform for native leaflet coaptation [18]. The Edwards PASCAL transcatheter mitral valve repair system utilizes features of both the FORMA and the MitraClip devices by combining a $10-\mathrm{mm}$ central spacer and two paddles that attach the device to the valve leaflets, theoretically providing better results for repair [18]. Another option that was used in one of our studies is surgical valve replacement to prevent TR [18].

Following the transcatheter tricuspid valve repair or replacement, the expected complications include acute kidney injury, bleeding events, in-hospital mortality, all-cause or cardiovascular related mortality, valve dysfunction, and device embolization. Atrial fibrillation is common in patients with severe TR, but the minimal development of new arrhythmias following treatment of the TR is promising [1]. Many patients with TR have extensive comorbidities: NYHA class 3 or 4 heart failure, diuretic use, history of renal impairment, hypertension, diabetes, chronic obstructive pulmonary disease, pulmonary hypertension, previous valve intervention, percutaneous coronary intervention or CABG, coronary artery disease, peripheral arterial diseases, and implanted PPMs or ICDs. In our analyses, the Fam et al. study revealed that two patients died $(7 \%$ mortality), one patient was hospitalized for a heart failure exacerbation and two patients had single leaflet attachment $(7 \%)$. No major bleeding events or cardiovascular events were noted (composite of nonfatal stroke, nonfatal myocardial infarction, and cardiovascular death). Lauten et al. revealed a $92 \%$ immediate procedural success, $8 \%$ device embolization, $12 \%$ bleeding complications, and mortality at 30 days was $12 \%$. Mehr et al. revealed $16.4 \%$ in-hospital mortality, MACE was $4.9 \%$, and all-cause mortality at 30 days was $4 \%$. Nickenig et. al in 2019 revealed cardiovascular mortality at 6 months to be around $4 \%$, major bleeding events at $11 \%$, and $7 \%$ single leaflet attachment. Nickening et al. also revealed that $97 \%$ of patients underwent successful deployment of the device, 5\% experienced new arrhythmias, 5\% had bleeding events which required transfusion and in-hospital mortality of $5 \%$. Perlman et al. revealed that mortality rate was $0 \%, 6 \%$ of patients had an infection and $6 \%$ had a TIA. Given that postoperative surgical mortality for patients with moderate or severe TR can be up to $80 \%$ [19], transcatheter approaches seem to have a relatively lower mortality rate and better side effect profile.

\section{Study Limitations}

Due to the novelty of tricuspid valve repair and replacement, we were only able to include 336 patients and 6 prospective controlled trials. This limits our ability to generalize the results to the general population. Our patients had higher risk profiles as well at baseline. These are also very early experiences and there needs to be more time for complete follow-up for our patients.
Follow-up was not uniform across all studies, with several studies missing important information such as hemodynamics, echocardiographic findings, etc. However, these were all prospective controlled trials highlighting a strength of our analysis.

\section{Conclusions}

Our pooled analysis shows that outcomes, specifically mortality and MACE, are minimal and much less than expected up to 1 year following transcatheter tricuspid valve repair or replacement. This favorable trend is promising for continuing to study the use of transcatheter tricuspid valve repair and valve replacement for moderate and severe TR. In future studies, a direct comparison of each device head-to-head would provide valuable insight into the impact on outcomes for tricuspid regurgitation for each device.

\section{Highlights}

- Tricuspid regurgitation is a poor prognostic marker of end-stage heart failure.

- Surgical Treatment options for severe tricuspid regurgitation are currently limited because these procedures have the highest mortality rates of all valve procedures.

- Transcatheter tricuspid valve repair or replacement (collectively, TVTT) are extremely promising interventions due to the minimally invasive nature of these treatments.

- Multiple devices are currently being developed and tested for TTVT to offer surgeons a safer alternative than the aforementioned traditionally high-risk surgical procedures.

- Six studies were included, 3 were registries (TriVALVE, TRILUMINATE, TRAMI), surveying a total of 336 patients. Complications following the procedures were pooled and analyzed.

- Despite a narrow sample size due to the novelty of these procedures and varying lengths of follow-up (30 days to 1 year), trans-catheter tricuspid valve repair and replacement prove to be promising interventions.

- Our pooled analysis shows that outcomes, specifically mortality and MACE, are minimal and much less than expected up to 1 year following transcatheter tricuspid valve repair or replacement.

- Mortality was significant at $14.6 \%$, but patients with severe tricuspid regurgitation have a poor prognosis overall without intervention along with higher comparative mortality rates.

- Research aimed at further investigating TVTT procedures and prospective clinical trials to establish these treatments as mainstays for severe tricuspid regurgitation is necessary. 


\section{Funding Information}

No funding or financial support has been allocated for this article.

\section{Disclosures}

All authors have no relevant disclosures with regards to this article.

\section{References}

1. Perlman G, Praz F, Puri R, Ofek H, Ye J, et al. (2017) Transcatheter tricuspid valve repair with a new transcatheter coaptation system for the treatment of severe tricuspid regurgitation: 1-Year Clinical and Echocardiographic Results. JACC Cardiovasc Interv 10: 1994-2003.

2. Oliveira DC, Oliveira CGC (2019) The forgotten, not studied or not valorized tricuspid valve: The transcatheter revolution is coming. Cardiol Res 10: 199-206.

3. Nickenig G, Weber M, Schueler R, Hausleiter J, Näbauer $\mathrm{M}$, et al. (2019) 6-month outcomes of tricuspid valve reconstruction for patients with severe tricuspid regurgitation. J Am Coll Cardiol 73: 1905-1915.

4. Nickenig G, Estevez-Loureiro R, Franzen O, Tamburino C, Vanderheyden M, et al. (2014) Percutaneous mitral valve edge-to-edge repair: In-hospital results and 1-year follow-up of 628 patients of the 2011-2012 Pilot European Sentinel Registry. J Am Coll Cardiol 64: 875-884.

5. Aboulhosn J, Cabalka AK, Levi DS, Himbert D, Testa L, et al. (2017) Transcatheter valve-in-ring implantation for the treatment of residual or recurrent tricuspid valve dysfunction after prior surgical repair. JACC Cardiovasc Interv 10: 53-63.

6. Nickenig G, Weber M, Lurz P, Bardeleben RS, Sitges M, et al. (2019) Transcatheter edge-to-edge repair for reduction of tricuspid regurgitation: 6-month outcomes of the TRILUMINATE single-arm study. Lancet 394: 2002-2011.

7. Guenther T, Noebauer C, Mazzitelli D, Busch R, Tassani-Prell $P$, et al. (2008) Tricuspid valve surgery: A thirty-year assessment of early and late outcome. Eur J Cardiothorac Surg 34: 402-409.

8. Taramasso M, Denti P, Buzzatti N, De Bonis M, La Canna G, et al. (2012) Mitraclip therapy and surgical mitral repair in patients with moderate to severe left ventricular failure causing functional mitral regurgitation: A single-centre experience. Eur J Cardiothorac Surg 42: 920-926.
9. Lauten A, Figulla HR, Unbehaun A, Fam N, Schofer J, et al. (2018) Interventional treatment of severe tricuspid regurgitation: Early clinical experience in a multicenter, observational, first-in-man study. Circ Cardiovasc Interv 11: e006061.

10. Fam NP, Ross HJ, Verma S (2016) Mitral clip - looking back and moving forward. Curr Opin Cardiol 31: 169-175.

11. Mehr M, Karam N, Taramasso M, Ouarrak T, Schneider S, et al. (2020) Combined tricuspid and mitral versus isolated mitral valve repair for severe MR and TR: An analysis from the trivalve and TRAMI registries. JACC Cardiovasc Interv 13: $543-550$

12. Campelo-Parada F, Perlman G, Philippon F, Ye J, Thompson C, et al. (2015) First-in-man experience of a novel transcatheter repair system for treating severe tricuspid regurgitation. J Am Coll Cardiol 66: 2475-2483.

13. Prihadi EA, Delgado V, Leon MB, Enriquez-Sarano M, Topilsky $Y$, et al. (2019) Morphologic types of tricuspid regurgitation: Characteristics and prognostic implications. JACC Cardiovasc Imaging 12: 491-499.

14. Moraca RJ, Moon MR, Lawton JS, Guthrie TJ, Aubuchon $\mathrm{KA}$, et al. (2009) Outcomes of tricuspid valve repair and replacement: A propensity analysis. Ann Thorac Surg 87: 83-88.

15. Wang N, Fulcher J, Abeysuriya N, McGrady M, Wilcox I, et al. (2019) Tricuspid regurgitation is associated with increased mortality independent of pulmonary pressures and right heart failure: A systematic review and meta-analysis. Eur Heart J 40: 476-484.

16. Taramasso $M$, Alessandrini $H$, Latib A, Asami M, Attinger-Toller A, et al. (2019) Outcomes after current transcatheter tricuspid valve intervention: Mid-term results from the International TriValve Registry. JACC Cardiovasc Interv 12: 155-165.

17. Rodés-Cabau J, Hahn RT, Latib A, Laule M, Lauten A, et al. (2016) Transcatheter therapies for treating tricuspid regurgitation. J Am Coll Cardiol 67: 1829-1845.

18. Gheorghe L, Rensing BJWM, Van der Heyden JAS, Eefting FD, Post MC, et al. (2019) Transcatheter tricuspid valve interventions: An emerging field. Curr Cardiol Rep 21: 37.

19. Hwang HY, Kim K-H, Kim K-B, Ahn H (2012) Mechanical tricuspid valve replacement is not superior in patients younger than 65 years who need long-term anticoagulation. Ann Thorac Surg 93: 1154-1160. 